\title{
CAPÍTULO 24: ETANOL 1,5G: UMA DISCUSSÃO SOBRE A INTEGRAÇÃO ENTRE O ETANOL DE PRIMEIRA E SEGUNDA GERAÇÃO
}

\section{CHAPTER 24: 1.5G ETHANOL: A DISCUSSION ABOUT THE COMBINATION BETWEEN FIRST AND SECOND GENERATION ETHANOL}

\author{
Vanessa Paola Miranda Zart ${ }^{1}$; Cristina Giatti Marques de Souza ${ }^{2}$; Rosane Marina Peralta ${ }^{3}$, Rafael \\ Castoldi $^{4}$
}

\begin{abstract}
Resumo
A produção de etanol a partir de fontes renováveis tem sido uma alternativa promissora na busca pela redução da taxa de emissão de dióxido de carbono para a atmosfera. Uma das principais fontes de obtenção de matéria prima para a produção do bioetanol é a cana-de-açúcar, predominantemente produzida no Brasil. A fim de otimizar a produção, tem sido proposta uma integração dos processos de produção do etanol de primeira e segunda gerações. $\mathrm{O}$ trabalho objetiva discutir os principais problemas relacionados a produção de etanol da segunda geração e apresentar as vantagens da integração dos processos. A pesquisa se dá por meio de uma revisão integrativa da literatura em conjunto com testes experimentais envolvendo o prétratamento biológico e produção de enzimas celulolíticas, evidenciando a eficácia do processo de produção 1,5G em melhorar o rendimento de etanol frente aos métodos autônomos de produção, conferindo assim uma maior viabilidade econômica mediante o compartilhamento de estruturas.
\end{abstract}

Palavras-Chave: Etanol, Bioetanol, 1,5G, Integração, Sacarificação, Cana-de-açúcar.

\begin{abstract}
The ethanol production from renewable sources has been a promising alternative in order to reduce the rate of carbon dioxide emissions into the atmosphere. One of the main sources of raw material for the bioethanol production is sugarcane, predominantly produced in Brazil. In order to optimize the production, an integration of the first and the second generation ethanol production processes has been proposed. The work aims to discuss the main problems related to second generation ethanol production and present the advantages of the integration of both processes. The research takes place through an integrative review of the literature, together with experimental tests involving biological pre-treatment and production of cellulolytic enzymes, showing the effectiveness of the $1.5 \mathrm{G}$ production process in improving ethanol yield, compared to autonomous methods of production and thus providing greater economic viability through the sharing of structures.
\end{abstract}

Keywords: Ethanol, Bioethanol, 1.5G, Integration, Sacarification, Sugarcane.

\footnotetext{
${ }^{1}$ Bioquímca, Universidade Estadual de Maringá (UEM), vanessa.zart@ gmail.com

${ }^{2}$ Profa. Dra., Universidade Estadual de Maringá (UEM), cgmsouza@uem.br

${ }^{3}$ Profa. Dra., Universidade Estadual de Maringá (UEM), rmperalta@uem.br

${ }^{4}$ Prof. Dr., Universidade Estadual de Maringá (UEM), rcastoldi@uem.br
} 


\section{Introdução}

Mudanças climáticas têm sido observadas com maior destaque nas últimas décadas, sendo, portanto, um alvo relevante de discussões políticas e científicas. O aquecimento global destaca-se em meio à tais mudanças, estando intimamente ligado ao aumento da emissão de dióxido de carbono $\left(\mathrm{CO}_{2}\right)$, considerado o principal gás responsável pelo denominado "efeito estufa" (GHG, do inglês greenhouse gas) (ANDERSON et al., 2016). A oxidação do carbono que ocorre durante a combustão de combustíveis fósseis tem sido uma das fontes predominantes de $\mathrm{CO}_{2}$ atmosférico (24\%), inferior apenas ao setor de eletricidade e geração de calor (Figura 1). Isso implica na ligação direta do setor de transportes aos impactos ambientais, uma vez que os combustíveis fósseis dominam mais da metade do mercado energético do setor (ADITIYA et al., 2016). Esses combustíveis ainda servem como o principal recurso global de energia e representam mais de $88 \%$ do consumo de energia primária, apesar de não serem provenientes de fontes renováveis e passíveis de esgotamento em um futuro próximo, de acordo com especialistas (LI, P. et al., 2019).

Figura 1. Gráfico de setores, indicando a porcentagem da emissão de $\mathrm{CO}_{2}$ proveniente da combustão de combustíveis fósseis por setor, no ano de 2015. *Em "outros" estão inclusos a agricultura/silvicultura, pesca, indústrias de energia que não sejam eletricidade e geração de calor, e outras emissões não especificadas em outros lugares.

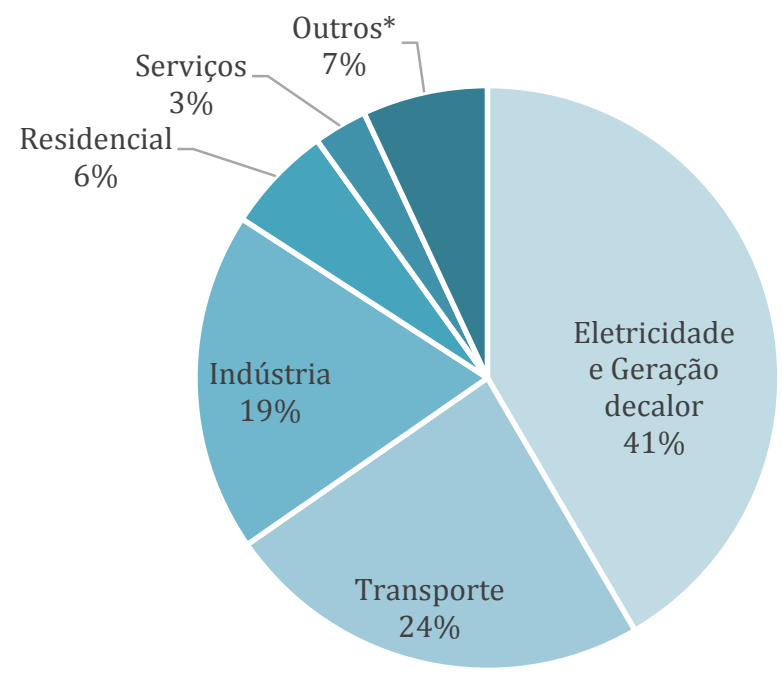

Fonte: Adaptado de International Energy Agency (2017).

Fontes de energia mais sustentáveis têm sido objetos fundamentais em pesquisas que objetivam a redução dos impactos ambientais causados pelos gases do efeito estufa. O bioetanol, produzido a partir de fontes renováveis, se apresenta como uma alternativa promissora para os combustíveis fósseis, podendo ser substituto total ou parcial da gasolina. No 
Brasil, a quantidade de álcool anidro presente na gasolina gira em torno de $23 \%$, segundo disposto pela Agência Nacional do Petróleo (ANP). No ano de 2019, o Brasil se mantém como o segundo país que mais produz etanol de maneira sustentável e eficiente, atrás apenas dos Estados Unidos. Juntos, os Estados Unidos e o Brasil produzem 84\% do etanol mundial, segundo o Departamento de Energia dos EUA (U.S. Department of Energy).

O etanol de primeira geração pode ser derivado do amido ou de outros açúcares por meio de um processo de fermentação. Uma das principais fontes de obtenção do açúcar proveniente de plantas utilizadas é a cana-de-açúcar, predominantemente produzida no Brasil. Esta cultura requer baixos recursos hídricos e terrestres por unidade de produção de energia biocombustível, além de emitir baixas quantidades de gases do efeito estufa (JIAO, J. et al., 2018). De acordo com Aditiya et al. (2016), uma das características mais favoráveis à utilização dessas culturas como matéria prima para a produção do etanol consiste no alto rendimento e em baixos custos de conversão, sendo os principais limitantes desse padrão de produção a sazonalidade do cultivo e seu conflito com a produção de finalidade alimentar.

Nesse contexto foi proposta uma segunda geração de bioetanol, tendo como foco a utilização de derivados de resíduos agrícolas e florestais como matérias-primas. Segundo Jambo et al. (2016), essa nova concepção minimiza o problema anteriormente citado, denominado "food versus fuel" (traduzindo do inglês para o português, "alimentos versus combustíveis"). Para a produção do bioetanol de segunda geração (2G), o açúcar é obtido através da conversão de materiais lignocelulósicos e amiláceos presentes nesses derivados. Tais açúcares podem ser posteriormente processados, obtendo como produto final o bioetanol anidro (ADITIYA et al., 2016). Por ser produzido a partir da biomassa, a segunda geração de etanol embasa uma fonte mais sustentável de energia, apresentando menores impactos ambientais em termos de emissões de $\mathrm{CO}_{2}$, se comparados aos combustíveis fósseis (HAJI ESMAEILI, S. A. et al., 2020). Existem, no entanto, dificuldades para a produção do bioetanol de segunda geração em larga escala, uma vez que o processo exige uma tecnologia mais cara e mais avançada. Além disso, apresenta altos custos de conversão e baixos rendimentos, o que é justificado pela presença da lignina presente em sua composição.

Dentro desse quadro, cientistas sugeriram a utilização de organismos marinhos, como as algas, na produção do bioetanol, surgindo assim a sua terceira geração. Algas são consideradas matérias-primas que apresentam um grande potencial de conversão da biomassa em energia. Contudo, a produção do bioetanol de terceira geração é dependente de diversos fatores como tecnologias avançadas e é dependente do ambiente marinho, dificultando sua viabilidade (JAMBO et al., 2016). 
Como alternativa aos problemas enfrentados pelas três gerações de bioetanol, foi proposta a integração dos processos de produção das duas primeiras gerações. O problema do baixo rendimento obtido pela produção de etanol lignocelulósico $(2 \mathrm{G})$, devido às baixas concentrações de açúcar após a hidrólise, é sanado facilmente pela primeira geração, a qual apresenta altas taxas de conversão.

Métodos de pré-tratamento utilizados na produção do etanol $2 \mathrm{G}$ geram uma grande variedade de compostos secundários, os quais atuam como inibidores para enzimas e outros microorganismos em etapas subsequentes. Com a integração dos processos, são necessárias concentrações mais baixas da matéria-prima lignocelulósica, levando a menores concentrações de inibidores formados durante o pré-tratamento, descartando a necessidade de desintoxicação (LENNARTSSON et al. 2014; BHATIA, S. K. et al. 2020).

Desta forma o presente estudo tem por objetivo discutir os atuais métodos de produção do bioetanol, com foco na integração entre os processos de primeira e segunda gerações e discutir os principais problemas na busca pela sua produção em larga escala. O trabalho também visa avaliar de que forma a produção de etanol da geração 1,5G pode ser considerada viável em território brasileiro, frente a produção do etanol de primeira e segunda geração.

\section{Desenvolvimento}

\section{A produção do bioetanol e fontes de biomassa}

$\mathrm{O}$ composto orgânico de fórmula molecular $\mathrm{C}_{2} \mathrm{H}_{6} \mathrm{O}$ é designado como etanol, álcool ou álcool etílico. A produção de etanol a partir de biomassa e fontes renováveis dá origem ao chamado bioetanol, pertencente à classe de biocombustíveis, dividindo espaço com o biodiesel, biobutanol, biogás, óleos vegetais e bioidrogênio. As principais matérias-primas são baseadas em produtos orgânicos com elevadas quantidades de carboidratos, usualmente açúcares de seis carbonos, passíveis de serem fermentados à etanol por enzimas fúngicas (NIGAM; SINGH, 2011).

Macromoléculas não podem ser diretamente convertidas a etanol por processos fermentativos convencionais, devendo primeiramente ser clivadas a moléculas mais simples, originando, por exemplo, a glicose. $\mathrm{O}$ amido, que consiste em um polímero formado por uma longa cadeia de glicose, apresenta-se como uma excelente matéria-prima na produção do bioetanol. Nos Estados Unidos (EUA) é amplamente utilizado o amido proveniente do milho, dada sua facilidade em ser degradado à glicose. No entanto, outras fontes de carboidratos são comumente utilizadas na produção do bioetanol, como por exemplo a cana-de-açúcar, a raiz de beterraba, frutas e suco de palmeira como fontes de açúcares; grãos como trigo, arroz, sorgo 
doce, milho e plantas de raiz como batata e mandioca como fontes de amido e também resíduos de madeira, cedro, pinho, resíduos agrícolas e fibras como fontes de celulose (ZABED et al., 2017).

A cana-de-açúcar é uma gramínea perene alta de cultura semi-perene e integrante da família Poaceae e do gênero Saccharum. A cana-de-açúcar é uma planta tropical, com múltiplos caules de aproximadamente 3 a 4 metros de altura e cinco centímetros de diâmetro. O caule constitui uma porção aproximada de $75 \%$ da planta quando madura, constituindo-se em 11-16\% de fibra, 12-16\% de açúcares solúveis, 2-3\% de não açúcares e 63-73\% de água. O cultivo da cana-de-açúcar é sensível ao clima, tipo de solo, irrigação, fertilizantes, insetos, controle de doenças, variedade da cana e época de colheita. É comumente cultivada em países tropicais e subtropicais e, portanto, muito bem adaptada ao clima brasileiro (MANOCHIO et al., 2017; CARVALHO et al., in press). Os principais constituintes do bagaço de cana são a celulose, hemicelulose e lignina. Um cálculo da composição realizado por Dias et al. (2012), com base em dados da literatura de aproximadamente 50 amostras, está representado graficamente na Figura 2, indicando a porcentagem de cada constituinte. Carvalho et al. (in press), aponta os valores de aproximadamente 41,3\% para celulose, 24,9\% para hemicelulose e 20,2\% para lignina. Extrativos e cinzas equivalem ambos a aproximadamente $4,9 \%$.

Figura 2. Composição do bagaço de cana-de-açúcar.

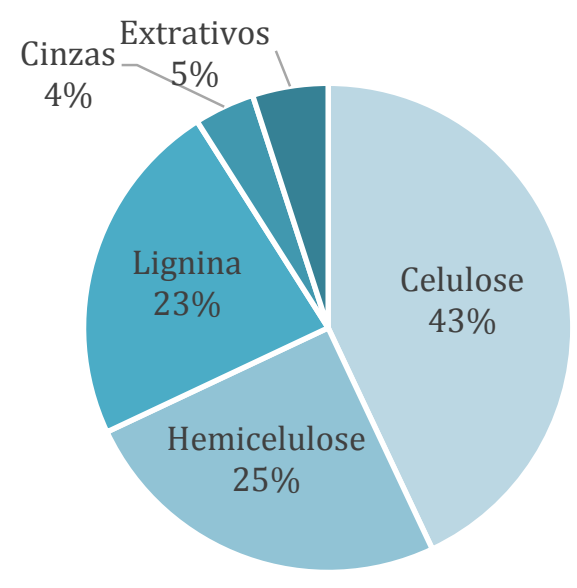

Fonte: Adaptado de Dias et al. (2012).

\section{Primeira geração do bioetanol}

A primeira geração de biocombustíveis líquidos é obtida a partir de açúcares, grãos ou sementes através de processos simples, até a formação do produto final. O processo 
convencional segue normalmente as etapas de recepção e limpeza da cana-de-açúcar, extração de açúcares, tratamento e concentração de suco, fermentação, destilação e desidratação. Após a recepção na fábrica, a cana deve ser limpa para remover parte da sujeira transportada pelo campo. A limpeza da cana-de-açúcar geralmente é feita com água de lavagem, que é reciclada para o processo de limpeza após a remoção de sujeira e outras impurezas. No processo de fermentação ocorre a conversão da glicose em etanol por leveduras e é interrompida ao atingir a concentração aproximada de $10 \%$ (v/v) de etanol, dado que concentração relativamente alta de etanol no meio de fermentação é desejável, contanto que não provoque inibição à levedura (VOHRA et al., 2014). A fermentação pode se dar de forma aeróbica ou anaeróbica, dependendo da disponibilidade e necessidade de oxigênio durante o processo.

As últimas etapas são a destilação e a desidratação, que resultam em uma solução azeotrópica de etanol a 95\% (v/v), podendo ser filtrada ou destilada até alcançar a concentração desejada de etanol hidratado ou anidro (VOHRA et al., 2014). O produto pode ser então misturado a combustíveis fósseis ou ser usado diretamente como combustível.

O bagaço, resíduo lignocelulósico do processamento de cana-de-açúcar, é queimado em caldeiras para gerar calor e eletricidade para a própria usina (cogeração) e caso haja excedente, pode ser exportado para o sistema elétrico nacional. Além de responder pela geração de energia, o bagaço da cana-de-açúcar também atrai atenção para sua utilização na produção do etanol de segunda geração. A simplificação do processo de produção do etanol de primeira geração (1G) está representada na Figura 3.

Figura 3. Fluxograma de blocos da simplificação do processo de produção do etanol de primeira geração, a partir do bagaço de cana.

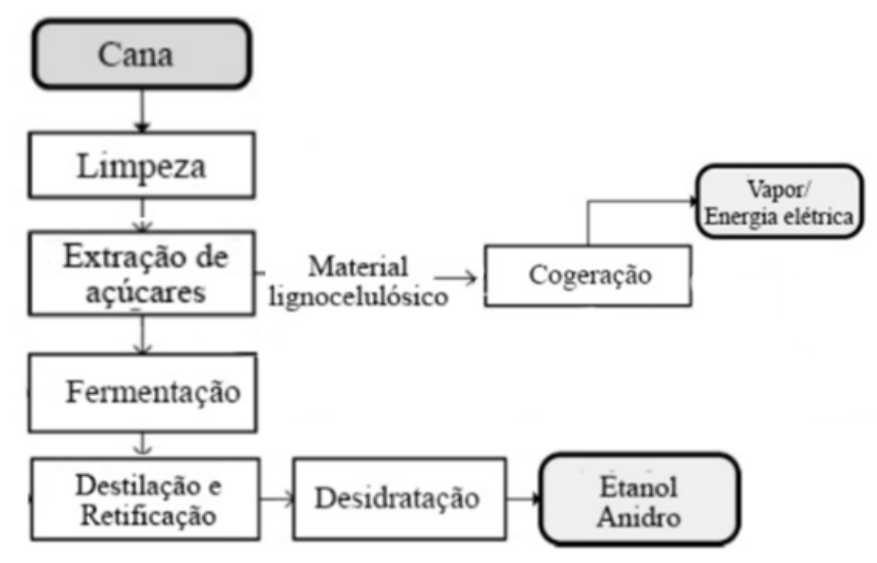

Fonte: Adaptado de (DIAS et al., 2013b). 


\section{Segunda geração do bioetanol}

No início dos anos 1970, quando a produção de etanol alavancou no Brasil, o bagaço era considerado estritamente um resíduo, o qual era eliminado por meio de caldeiras de baixa eficiência. Entretanto, com o passar do tempo, passou a ser reconhecido como uma matériaprima promissora na produção de etanol celulósico ou de segunda geração (2G), reduzindo os impactos ao meio ambiente.

A biomassa lignocelulósica atua como matéria-prima na produção do bioetanol 2G, sendo composta principalmente de celulose, hemicelulose e lignina (SINDHU et al., 2016). Porém, esse complexo forma uma barreira física, conferindo resistência, rigidez e impermeabilidade, dificultando o acesso de microrganismos. Para o uso da biomassa lignocelulósica na produção de etanol, quatro etapas são essenciais, sendo elas o pré-tratamento, hidrólise enzimática, fermentação dos polímeros de açúcares em etanol e destilação (RASTOGI; SHRIVASTAVA, 2017). O pré-tratamento tem papel fundamental na conversão da matéria-prima ao etanol, promovendo a despolimerização da lignocelulose. A estrutura cristalina da celulose é um dos principais fatores que dificultam a hidrólise e, portanto, o processo tem a finalidade de reduzir o seu grau de cristalinidade, favorecendo a forma amorfa, a qual é mais adequada ao ataque enzimático.

Em suma, os principais objetivos do processo de pré-tratamento são a redução de tamanho físico do material, a exposição dos componentes (hemicelulose, celulose e lignina) antes da hidrólise para melhorar a produção açúcares redutores, a viabilização do acesso às enzimas para a hidrólise dos carboidratos em soluções fermentáveis e a redução do grau de cristalinidade da matriz de celulose.

\section{Composição da biomassa e enzimas}

Os materiais lignocelulósicos são os principais componentes da biomassa e representam o recurso de energia renovável mais abundante disponível na terra (LORENCI WOICIECHOWSKI, A. et al. 2020), podendo amenizar o conflito entre o uso de alimentos e energia. Resíduos agrícolas contêm uma grande fração de substâncias lignocelulósicas e a abundância relativa desses três componentes difere para cada tipo de biomassa (PAUDEL et al., 2017).

Dentre essas porções, a lignina torna-se um dos maiores entraves. Para degradar essa estrutura são utilizadas enzimas, como a lignina peroxidase (LiP), a manganês peroxidase $(\mathrm{MnP})$ e a lacase, enzimas oxidativas provenientes dos fungos da podridão branca (WAN; LI, 2012), responsáveis pela deslignificação (redução do teor de lignina) e desintoxicação (remoção 
de compostos químicos da hidrolise de açúcar) da biomassa lignocelulósica. (RASTOGI; SHRIVASTAVA, 2017).

As enzimas atuam de forma a fornecer fontes de carbono mais facilmente digeríveis para o crescimento e metabolismo dos fungos. A hidrólise enzimática é o passo crítico para a produção de bioetanol, onde os carboidratos complexos são convertidos em monômeros simples (GUPTA; VERMA, 2015). Como as enzimas microbianas são eficientes na degradação de compostos lignocelulósicos, sabe-se que o pré-tratamento biológico é adequado para biomassa contendo lignoceluloses, embora a sua taxa de reação enzimática seja lenta e os custos mais elevados se comparado a outros métodos (PAUDEL et al., 2017). As estruturas referentes às unidades estruturais da celulose e lignina estão representadas na Figura 4.

Figura 4. Representação esquemática de (a) estrutura da celulose e (b) estrutura da lignina.
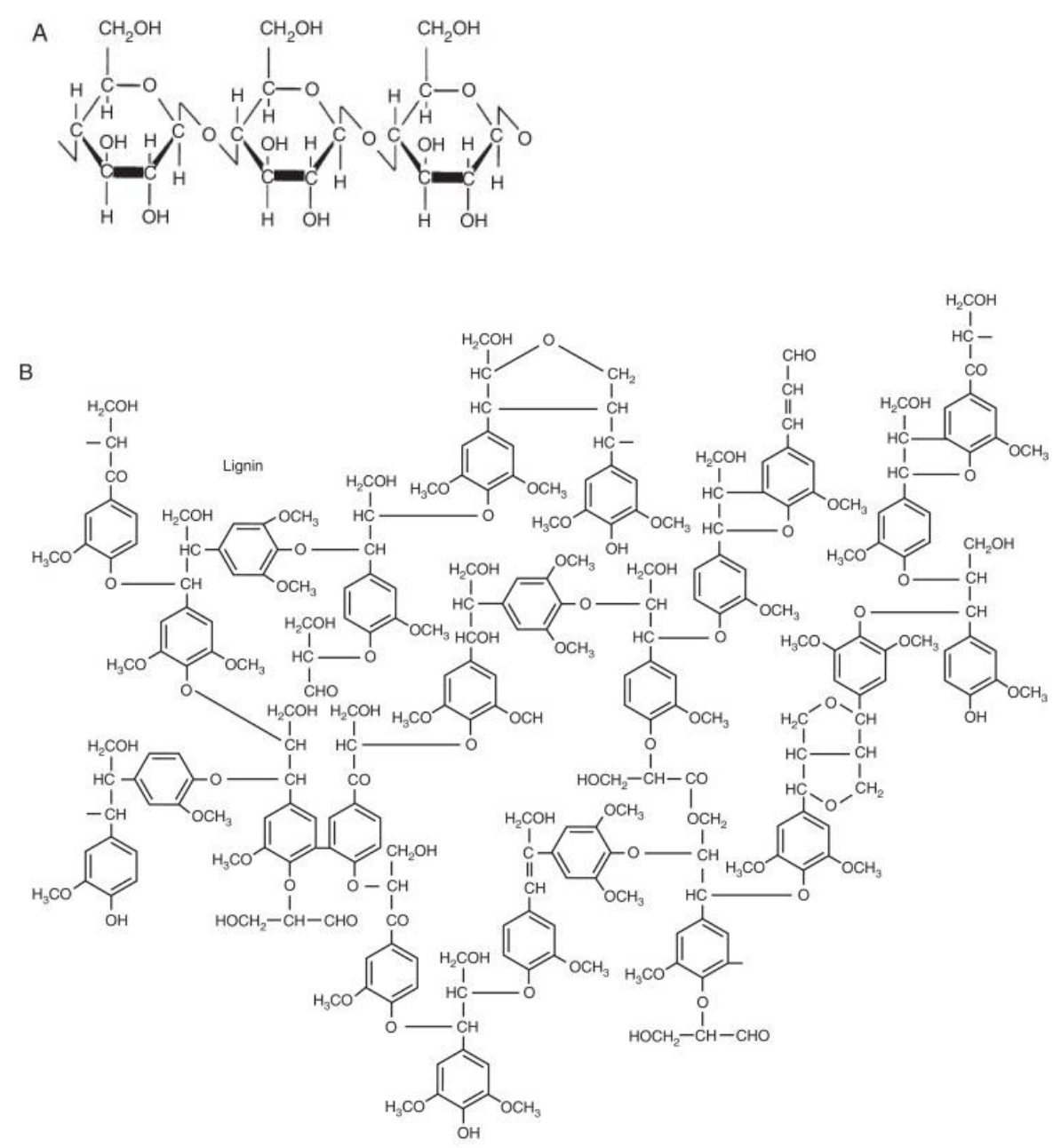

Fonte: Extraído de Watkins et al. (2014).

Um procedimento adequado para a degradação desses componentes é de fundamental importância para o desenvolvimento de novas tecnologias, visando principalmente a otimização 
do processo de produção do etanol de segunda geração, o qual pode se dar de forma mais eficiente pela integração com o processo de produção de etanol de primeira geração, resultando na promissora geração 1,5 .

\section{Eficiência do pré-tratamento biológico frente a outros métodos}

A eficiência de remoção de lignina depende do tipo de pré-tratamento empregado e das condições ideais mantidas durante o pré-tratamento. Os processos de pré-tratamento podem ser físicos, químicos, biológicos ou podem ser uma combinação desses métodos (NIJU, S.; SWATHIKA, M., 2019). Os pré-tratamentos físicos se baseiam no aumento da área superficial e no volume de poros acessíveis, diminuindo o grau de polimerização da celulose e sua cristalinidade, promovendo a hidrólise das hemiceluloses e despolimerização parcial da lignina (ZABED et al., 2016). Pré-tratamentos químicos e físico-químicos, tais como explosão a vapor, utilização de ácidos e bases, oxidação, radiação ionizante e utilização de amônia e solventes orgânicos também causam a desestabilização da parede celular, a solubilização da lignina e outras respostas necessárias antes da hidrólise enzimática (WANDERLEY et al., 2013; RABEMANOLONTSOA; SAKA, 2016; DA SILVA et al., 2016).

O pré-tratamento biológico baseia-se na capacidade de determinados organismos em degradar a lignina para obter acesso a celulose e hemicelulose. Os microrganismos mais utilizados são os fungos das podridões branca, parda e mole, além de algumas bactérias ruminantes, sendo que os da podridão branca são considerados mais eficientes para este fim (WAN; LI, 2012), uma vez que consomem menos energia e causam menos danos ao meio ambiente. Diversos fatores influenciam na degradação biológica da lignocelulose, como tensão fúngica, condições de cultura, secreção enzimática e mecanismos oxidativos (SALVACHÚA et al., 2011).

Vários estudos revelaram que o pré-tratamento biológico, especialmente utilizando fungos da podridão branca, é capaz de melhorar a eficiência da hidrólise com menor gasto de energia, de modo que a conversão da fração de carboidratos para açúcares monoméricos possa ser alcançada mais rapidamente e com maiores rendimentos (SINDHU; BINOD; PANDEY, 2016). Um dos maiores benefícios do pré-tratamento biológico é que o subproduto resultante deste método não inibe a hidrólise subsequente, bem como a fermentação, uma vez que é realizado em condições moderadas. É um processo que dispensa a reciclagem química e não libera nenhum composto perigoso ou tóxico para o meio ambiente (UMMALYMA, S. B. et al., 2019). 
Segundo SINDHU et al. (2016), rendimentos de até $82 \%$ no processo de sacarificação foram obtidos após pré-tratamentos biológicos, utilizando o fungo da podridão branca, Irpex lacteus sobre talos de milho. Apesar do alto rendimento, estes resultados foram obtidos no vigésimo oitavo dia de pré-tratamento, apontando uma das principais limitações do método biológico, isto é, o tempo.

O pré-tratamento biológico apresenta várias vantagens sobre outros métodos de prétratamento, sendo a principal desvantagem dessa estratégia o processo lento, bem como o fato de que os açúcares podem ser usados pelos microrganismos para seu crescimento. No entanto, enefícios ao meio ambiente devem ser destacados, para que novas pesquisas possibilitem a viabilidade de tal método de pré-tratamento, através da otimização do processo e redução do o tempo, crucial para seu uso tecnológico.

\section{Breve abordagem ao cenário brasileiro de produção de etanol e principais limitações}

Os combustíveis de primeira geração são produzidos atualmente em larga escala em diversos países. O maior questionamento envolvendo esse tipo de produção está relacionado ao conflito com a oferta de alimentos. O baixo aproveitamento da biomassa total das plantas reduz a eficiência do uso da terra e essa concorrência com a produção de alimentos implica num alto custo de produção. Esses custos podem variar de acordo com a matéria-prima utilizada, processo de conversão, escala de produção e região. O cultivo acessível da cana-de-açúcar no Brasil torna a produção do bioetanol uma opção viável. Pelo fato de a planta ser produzida em volumes substanciais, o etanol à base de cana-de-açúcar está se tornando uma alternativa cada vez mais econômica aos combustíveis fósseis. Isso incentiva a busca por novas tecnologias de obtenção de melhores rendimentos em seu processo de fabricação.

Segundo aponta a revista Novacana, no ano de 2007 o Brasil contava com 370 unidades de processamento da cana-de-açúcar, das quais 355 se voltavam à produção de etanol. O rendimento médio de usinas mistas se encontra em torno de $71 \mathrm{~kg}$ açúcar e 42 litros de etanol por tonelada de cana processada. A distribuição das usinas sucroalcooleiras em território brasileiro no ano de 2015, de acordo com seu tipo, pode ser observada na Figura 5. 
Figura 5. Produção de etanol por tipo, por Unidade da Federação em território brasileiro no ano de 2015.

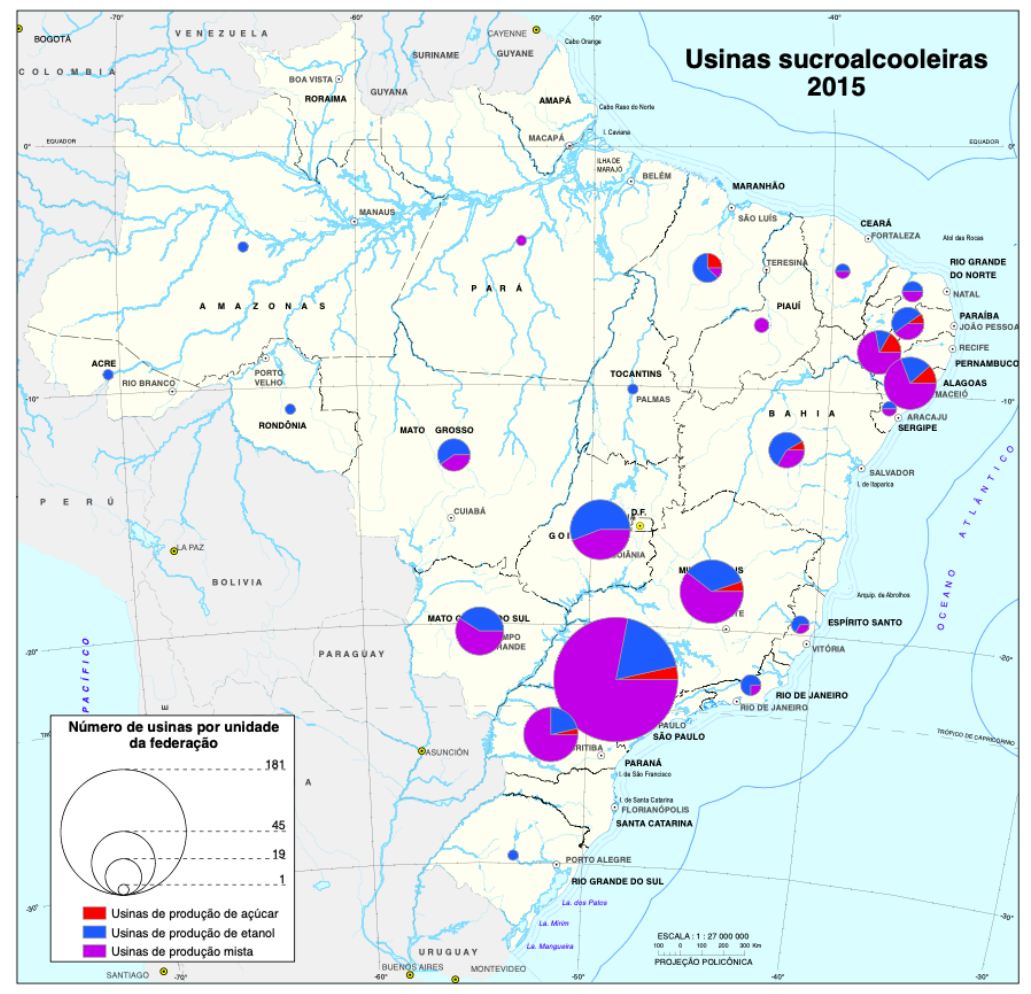

Fonte: Extraído de IBGE (2017).

\section{Atuais limitações na produção de bioetanol de segunda geração}

Os principais fatores que limitam a comercialização e produção em larga escala do etanol de segunda geração são o custo, bem como a baixa eficiência hidrolítica das enzimas no processo de sacarificação, bem como o tempo demandado em caso de pré-tratamento biológico. $\mathrm{O}$ dispêndio mais influente na produção de etanol $2 \mathrm{G}$ é baseada na aquisição de enzimas, seguido preço de venda da eletricidade produzida e em menor escala, o custo de transporte de folhas e outros materiais remanescentes da colheita da cana-de-açúcar (MACRELLI et al., 2014).

Portanto, os esforços atuais visam essencialmente potencializar a eficiência de produção, principalmente através da combinação de tecnologias e intensificação do aproveitamento de resíduos remanescentes, para que assim o processo possa ser economicamente viável e ambientalmente sustentável (ZABED et al., 2016).

\section{Geração 1,5 de etanol}

O desenvolvimento de projetos e pesquisas relacionadas ao etanol de segunda geração tem apresentado declínio devido aos problemas apresentados e ao limitado avanço nas usinas 
em funcionamento. Em contraposição, uma produção mais competitiva do etanol $2 \mathrm{G}$ pode ser alcançada mediante a integração das tecnologias $1 \mathrm{G}$ e $2 \mathrm{G}$ em uma usina, compartilhando fluxos de energia e materiais em unidades de operação, além de permitir o aproveitamento mais eficiente das fontes alternativas para produção de combustível.

O rendimento de etanol da cana-de-açúcar pode ser aumentado se o bagaço, já presente no local como resíduo de processo de produção do etanol de primeira geração, for tratado para produzir etanol $2 \mathrm{G}$, ao invés de seguir para a queima na produção de eletricidade, embora a rentabilidade seja fortemente dependente do preço de etanol. A utilização de todo o material na produção de subprodutos pode apresentar um impacto considerável sobre a viabilidade econômica da integração (MACRELLI et al., 2012).

Ou seja, a execução integrada dos processos de produção requer menores investimentos se comparada a uma usina autônoma, uma vez que algumas operações (como por exemplo a concentração, fermentação, destilação, armazenamento e cogeração) podem ser compartilhadas entre as duas plantas. Por já estarem disponíveis na própria usina ou próximo a ela, os resíduos da produção de etanol $1 \mathrm{G}$ são mais facilmente aproveitados para a produção de bioetanol 1,5G.

É importante notar que o uso da biomassa lignocelulósica para produzir o etanol 1,5G pode impedir a competição futura pelo uso da terra entre a produção de etanol e a produção de alimentos, possibilitando uma ampliação no rendimento de etanol sem interferir na área de produção alimentícia (CARPIO; SIMONE DE SOUZA, 2017).

Além disso, os problemas relacionados à formação de inibidores de fermentação durante o prétratamento terão efeitos inferiores nos rendimentos, uma vez que o hidrolisado será fermentado e acrescido ao caldo de cana-de-açúcar, diluindo esses inibidores. A possível recuperação do solvente na deslignificação alcalina pode, conjuntamente, apresentar inúmeros benefícios num aspecto ambiental (DIAS et al., 2012a).

\section{Integração dos processos de produção}

As principais etapas do processo na produção de etanol $1 \mathrm{G}$ são a moagem da cana de açúcar e a fermentação dos açúcares disponíveis. A produção de etanol $2 \mathrm{G}$ é, no entanto, mais complexa, devido a necessidade de um pré-tratamento a fim de decompor a matriz estrutural e clivar os açúcares poliméricos a monômeros fermentáveis, antes da conversão microbiana ao etanol. Os principais desafios neste processo são maximizar a conversão de lignocelulose e minimizar a perda de açúcares, o que é difícil usando condições operacionais amenas.

A integração é possível em várias etapas do processo, desde o pré-tratamento, até os processos posteriores, como por exemplo, nas etapas de destilação ou evaporação. Assim, 
diferentes configurações de processos são possíveis. Dois principais métodos de integração são a integração no estágio de fermentação ou no estágio de cultivo de fungos. Em ambos os casos, a primeira geração praticamente não sofre alterações, sendo um pouco mais evidente na primeira opção, pois os inibidores do processo da segunda geração não podem ser inseridos nos fermentadores (LENNARTSSON et al., 2014).

Um dos principais coprodutos da produção de etanol a partir da cana-de-açúcar e do bagaço é a eletricidade. Ao projetar uma usina integrada, uma das duas principais configurações pode ser escolhida: a produção de biogás ou a produção de DDGS (Grãos secos destilados com solúveis ou Dried Distillers Grains with Solubles, em inglês), derivados do milho, para a utilização na nutrição de monogástricos. Representações esquemáticas de ambos os processos de produção podem ser observadas nas Figura 6 (a) e (b). A escolha depende principalmente do mercado de DDGS e biogás no local da usina. No entanto, segundo aponta Dias et al. (2012), a produção de biogás manifesta uma menor notoriedade enquanto não oferecer qualidade de combustível para veículos, dada a baixa aplicação. A grande produção de DDGS e a fermentação combinada de pentoses e hexoses tem sido mais promissoras, se comparadas à produção de biogás. Além disso, caso a legislação impeça a produção de DDGS que incluem leveduras geneticamente modificadas, será necessária uma etapa de separação antes da fermentação de pentoses e hexoses no processo $1 \mathrm{G}$, para separar os sólidos da fração líquida, a fim que estes sólidos possam ser utilizados para a produção de DDGS sem serem misturados com a levedura (JOELSSON et al., 2016).

Figura 6. Fluxograma de blocos da integração entre os processos de produção da primeira e segunda geração de etanol a partir do bagaço de cana. (a) representa a produção de DDGS e (b) a produção de biogás.

(a)

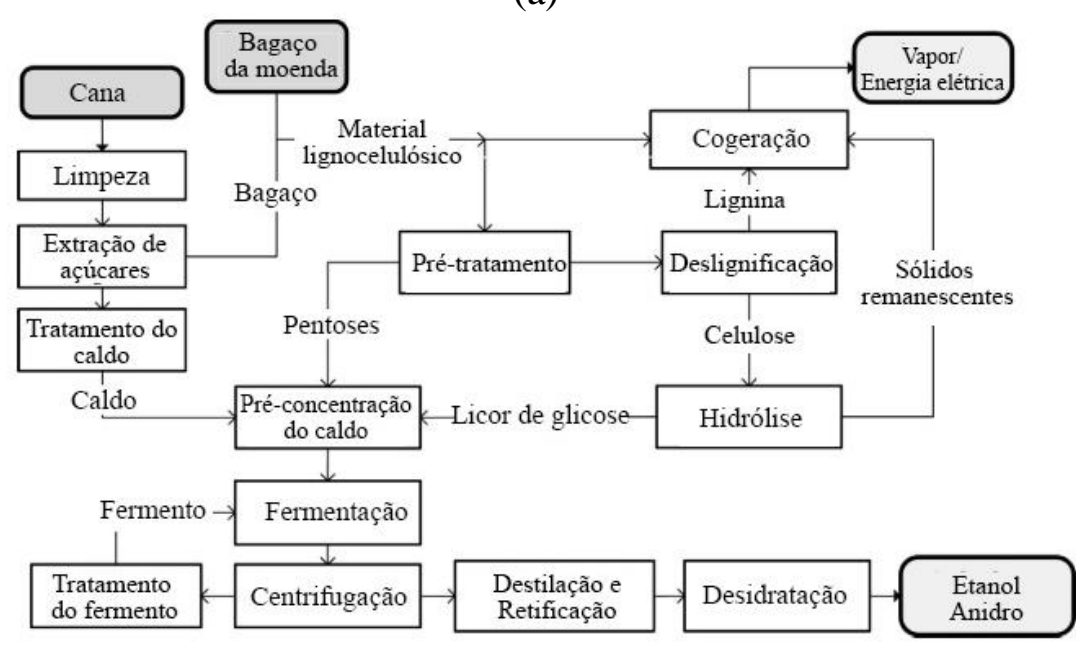


(b)

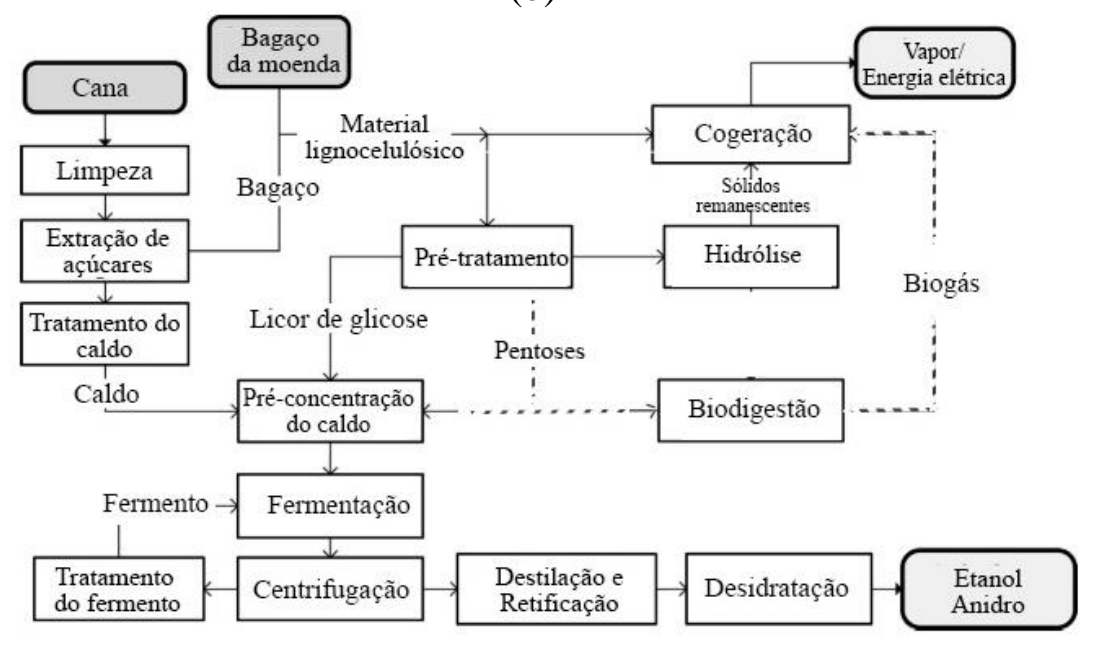

Fonte: Adaptado de (DIAS et al., 2013b) e (DIAS et al., 2013c).

Em se tratando do processo de produção que visa a obtenção de DDGS, a escolha de produtos químicos para o pré-tratamento também se torna crucial. No entanto, o enxofre possui limitações em relação à alimentação animal, podendo demandar a ausência do ácido sulfúrico no pré-tratamento das lignoceluloses. A hidrólise é comumente realizada com a utilização de enzimas, podendo ocorrer em compartimentos separados ou juntos à fermentação, sem apresentar significativa influência na qualidade de DDGS. Após a hidrólise, as hexoses liberadas serão convertidas em etanol e $\mathrm{CO}_{2}$ pelo microrganismo fermentador, como de costume.

Segundo estudos guiados por Dias et al. (2013), pôde-se verificar que a fermentação das pentoses leva a uma maior produção de etanol, mas a quantidade de material lignocelulósico hidrolisado é menor do que nas configurações onde as pentoses são biodigeridas, infelizmente com maior demanda de energia das etapas de concentração e destilação, devido ao aumento na produção de etanol. Contudo, como a concentração elevada de etanol no meio de fermentação é desejável, para atingir um menor consumo de energia na etapa de purificação, a concentração de açúcares no líquido hidrolisado desempenha um papel importante no dado processo.

Recuperar parte dos resíduos do campo pode aumentar a quantidade de material lignocelulósico excedente disponível para hidrólise, uma vez que estes podem ser queimados para fornecer energia para suprir as necessidades do processo industrial (DIAS et al., 2012b). A redução do consumo de vapor e a maximização da qualidade do material lignocelulósico excedente é fundamental para aumentar o rendimento de usinas que visam a utilização do bagaço e dos resíduos resultantes na produção de etanol 2G. Além disso, pentoses podem também ser fermentadas até etanol, aumentando a produção de etanol do processo. No entanto, 
esta prática implica também em um aumento no consumo de energia (DIAS et al., 2012a). Na integração, a biomassa fúngica também pode ser utilizada para outros fins. Algumas cepas produzem lipídeos que podem ser extraídos da biomassa e vendidos como, por exemplo, suplementos dietéticos (BELLOU et al., 2012).

O uso dessa tecnologia tem chamado a atenção para o futuro desenvolvimento de biocombustíveis, principalmente no Brasil, pela otimização da estrutura de centenas de usinas de primeira geração, no país onde a produção de bioetanol em larga escala já é realidade.

\section{Próximos desafios na produção do etanol}

Um dos maiores desafios do processo de produção de etanol lignocelulósico está na obtenção de concentrações suficientemente altas de açúcar após a hidrólise. De um modo geral, o presente problema pode ser resolvido mediante a integração entre os processos de produção de etanol $1 \mathrm{G}$ e $2 \mathrm{G}$, uma vez que concentrações suficientemente altas são facilmente alcançadas na primeira geração. Assim, são necessárias concentrações mais baixas da matéria-prima lignocelulósica, levando a uma diminuição na concentração de inibidores formados durante o pré-tratamento, resolvendo consequentemente a necessidade de desintoxicação.

Segundo aponta Lennartson el al. (2014), o problema relacionado à utilização de pentoses pode ser resolvido através da utilização de cepas geneticamente modificadas de $S$. cerevisiae. Entretanto, a opinião pública negativa pode se tornar um problema. Outros microrganismos capazes de fermentar pentoses em etanol também poderiam ser empregados, mas geralmente são bastante sensíveis a inibidores (inclusive ao etanol). As pentoses também poderiam ser aplicadas na produção de compostos diferentes do etanol em etapas posteriores do processo. Porém, a dedicação e adequação do processo para tal aproveitamento não seria economicamente ideal, devido às baixas concentrações produzidas.

A incrustação, em particular, e a viscosidade do líquido também podem ser grandes obstáculos no processo. Ao reduzir a quantidade total de sólidos suspensos e compostos orgânicos no líquido, a gravidade desses obstáculos pode ser reduzida. Isso poderia permitir que mais água fosse removida nos evaporadores e menos nos secadores. Poderia também permitir que mais da vinhaça fina fosse enviada de volta ao processo, o que diminuiria diretamente a carga nos evaporadores e nos secadores (LENNARTSSON et al., 2014).

De um modo geral, a produção integrada de etanol de primeira e segunda gerações apresenta várias vantagens sobre as usinas independentes de produção de etanol, sem, no entanto, abnegar inúmeros aspectos a serem melhorados para que a nova geração de combustíveis renováveis possa ser realidade no Brasil. 


\section{Considerações Finais}

O desenvolvimento sustentável fornece uma base para a diminuição dos efeitos que levam a mudanças climáticas e o emprego da bioenergia tende a impactar positivamente na mitigação desses efeitos. Em se tratando da produção de bioetanol a partir da cana-de-açúcar, principal fonte de açúcar para fermentação em território brasileiro, foram avaliados os atuais métodos de produção, além da realização de um pré-tratamento biológico para a sacarificação do bagaço de cana.

A comparação dos atuais métodos de produção do bioetanol permitiu constatar uma maior viabilidade econômica na integração dos processos de produção $1 \mathrm{G}$ e $2 \mathrm{G}$ em virtude do compartilhamento estrutural e de equipamentos, podendo se dar em diferentes etapas do processo, amplificando sua versatilidade e aplicação. A indesejável geração de subprodutos devido à degradação da lignocelulose pode ser amenizada ao diluir o hidrolisado ao caldo de cana e os resíduos da colheita podem ser recuperados e aproveitados, evidenciando os aspectos positivos da integração.

Com um pré-tratamento biológico adequado e eficiente aliado à integração dos processos de produção, o rendimento de etanol pode ser potencializado, trazendo inúmeros benefícios econômicos e ao meio ambiente. Com base nos resultados, pode-se enfatizar a importância da avaliação do processo de produção de bioetanol para uma compreensão mais clara, orientando à novas pesquisas capazes de preencher as lacunas e expandir os benefícios da integração do etanol de primeira e segunda geração.

\section{Referências}

ADITIYA, H. B. et al. Second generation bioethanol production: A critical review. Renewable and Sustainable Energy Reviews, v. 66, p. 631-653, 2016.

ADSUL, M. G. et al. Polysaccharides from bagasse : applications in cellulase and xylanase production. v. 57, p. 67-72, 2004.

ANDERSON, T. R.; HAWKINS, E.; JONES, P. D. CO2, the greenhouse effect and global warming: from the pioneering work of Arrhenius and Callendar to today's Earth System Models. Endeavour, v. 40, n. 3, p. 178-187, 2016.

BELLOU, S. et al. Lipids Containing Polyunsaturated Fatty Acids Synthesized by Zygomycetes Grown on Glycerol. p. 146-158, 2012.

BHATIA, S. K. et al. Recent developments in pretreatment technologies on lignocellulosic biomass: Effect of key parameters, technological improvements, and challenges. Bioresource Technology, v. 300, n. January, p. 122724, 2020.

CARPIO, L. G. T.; SIMONE DE SOUZA, F. Optimal allocation of sugarcane bagasse for 
producing bioelectricity and second generation ethanol in Brazil: Scenarios of cost reductions. Renewable Energy, v. 111, p. 771-780, 2017.

CARVALHO, D. J. et al. (in press). Assessment of the self-sustained energy generation of an integrated first and second generation ethanol production from sugarcane through the characterization of the hydrolysis process residues. Energy Conversion and Management, v. 203, n. November, p. 112267, 2020. (Prevision screen - November/2020)

DA SILVA, A. R. G.; TORRES ORTEGA, C. E.; RONG, B. G. Techno-economic analysis of different pretreatment processes for lignocellulosic-based bioethanol production. Bioresource Technology, v. 218, p. 561-570, 2016.

DANTAS, G. A.; LEGEY, L. F. L.; MAZZONE, A. Energy from sugarcane bagasse in Brazil: An assessment of the productivity and cost of different technological routes. Renewable and Sustainable Energy Reviews, v. 21, p. 356-364, 2013.

DEMIRBAS, A. Biofuels securing the planet's future energy needs. Energy Conversion and Management, v. 50, n. 9, p. 2239-2249, 2009.

DIAS, M. O. S. et al. Improving second generation ethanol production through optimization of first generation production process from sugarcane. Energy, v. 43, n. 1, p. 246-252, 2012a.

DIAS, M. O. S. et al. Integrated versus stand-alone second generation ethanol production from sugarcane bagasse and trash. Bioresource Technology, v. 103, n. 1, p. 152-161, 2012b.

DIAS, M. O. S. et al. Evaluation of process configurations for second generation integrated with first generation bioethanol production from sugarcane. Fuel Processing Technology, v. 109, p. 84-89, 2013 a.

DIAS, M. O. S. et al. Biorefineries for the production of first and second generation ethanol and electricity from sugarcane. Applied Energy, v. 109, p. 72-78, 2013 b.

DIAS, M. O. S. et al. Cogeneration in integrated first and second generation ethanol from sugarcane. Chemical Engineering Research and Design, v. 91, n. 8, p. 1411-1417, 2013c.

GUPTA et al. Evaluation of pretreatment methods in improving the enzymatic saccharification of cellulosic materials. Carbohydrate Polymers, New Delhi, v. 84, n. 3, p. 1103-1109, 17 mar. 2011.

GUPTA, A.; VERMA, J. P. Sustainable bio-ethanol production from agro-residues: A review. Renewable and Sustainable Energy Reviews, v. 41, p. 550-567, 2015.

HAJI ESMAEILI, S. A. et al. First-generation vs. second-generation: A market incentives analysis for bioethanol supply chains with carbon policies. Applied Energy, v. 277, n. July, p. $115606,2020$.

IBGE. Dinâmica Territorial da Produção Agropecuária, 2017. Disponível em: <https://www.ibge.gov.br/apps/dinamica_agropecuaria/>. Acesso em 2 de set. de 2020

JAMBO, S. A. et al. A review on third generation bioethanol feedstock. Renewable and Sustainable Energy Reviews, v. 65, p. 756-769, 2016. 
JEYA, M. et al. Bioresource Technology Enhanced saccharification of alkali-treated rice straw by cellulase from Trametes hirsuta and statistical optimization of hydrolysis conditions by RSM. Bioresource Technology, v. 100, n. 21, p. 5155-5161, 2009.

JIAO, J.; LI, J.; BAI, Y. Ethanol as a vehicle fuel in China: A review from the perspectives of raw material resource, vehicle, and infrastructure. Journal of Cleaner Production, v. 180, p. 832-845, 2018.

JOELSSON, E. et al. Techno-economic evaluation of integrated first- and second-generation ethanol production from grain and straw. Biotechnology for Biofuels, v. 9, n. 1, p. 1-16, 2016.

KAPARAJU, P. et al. Bioresource Technology Bioethanol, biohydrogen and biogas production from wheat straw in a biorefinery concept. Bioresource Technology, v. 100, n. 9, p. 25622568, 2009.

LENNARTSSON, P. R.; ERLANDSSON, P.; TAHERZADEH, M. J. Integration of the first and second generation bioethanol processes and the importance of by-products. Bioresource Technology, v. 165, n. C, p. 3-8, 2014.

LI, P.; SAKURAGI, K.; MAKINO, H. Extraction techniques in sustainable biofuel production: A concise review. Fuel Processing Technology, v. 193, n. May, p. 295-303, 2019.

LORENCI WOICIECHOWSKI, A. et al. Lignocellulosic biomass: acid and alkaline pretreatments and their effects on biomass recalcitrance - conventional processing and recent advances. Bioresource technology, v. 304, n. January, p. 122848, 2020.

MACRELLI, S.; GALBE, M.; WALLBERG, O. Effects of production and market factors on ethanol profitability for an integrated first and second generation ethanol plant using the whole sugarcane as feedstock. Biotechnology for Biofuels, v. 7, n. 1, p. 1-16, 2014.

MACRELLI, S.; MOGENSEN, J.; ZACCHI, G. Techno economic evaluation of 2nd generation bioethanol production from sugar cane bagasse and leaves integrated with the sugar based ethanol process. Biotechnology for Biofuels, v. 5, p. 22, 2012.

MANOCHIO, C. et al. Ethanol from biomass: A comparative overview. Renewable and Sustainable Energy Reviews, v. 80, n. February, p. 743-755, 2017.

MILLER, G. A. I. L. Use of Dinitrosalicylic Acid Reagent for Detection of Reducing Sugars. Analytical Chemistry - ANAL CHEM, v. 31, 1959.

NAIK, S. N. et al. Production of first and second generation biofuels: A comprehensive review. Renewable and Sustainable Energy Reviews, v. 14, n. 2, p. 578-597, 2010.

NIJU, S.; SWATHIKA, M. Delignification of sugarcane bagasse using pretreatment strategies for bioethanol production. Biocatalysis and Agricultural Biotechnology, v. 20, n. May, p. $101263,2019$.

NIGAM, P. S.; SINGH, A. Production of liquid biofuels from renewable resources. Progress in Energy and Combustion Science, v. 37, n. 1, p. 52-68, 2011.

PAUDEL, S. R. et al. Pretreatment of agricultural biomass for anaerobic digestion: Current 
state and challenges. Bioresource Technology, v. 245, n. August, p. 1194-1205, 2017.

RABELO, S. C. et al. Production of bioethanol, methane and heat from sugarcane bagasse in a biorefinery concept. Bioresource Technology, v. 102, n. 17, p. 7887-7895, 2011.

RABEMANOLONTSOA, H.; SAKA, S. Various pretreatments of lignocellulosics. Bioresource Technology, v. 199, p. 83-91, 2016.

RAMESH, T. et al. A genetic model of amyotrophic lateral sclerosis in zebrafish displays phenotypic hallmarks of motoneuron disease. v. 662, p. 652-662, 2010.

RASTOGI, M.; SHRIVASTAVA, S. Recent advances in second generation bioethanol production: An insight to pretreatment, saccharification and fermentation processes. Renewable and Sustainable Energy Reviews, v. 80, n. May, p. 330-340, 2017.

SALVACHÚA, D. et al. Fungal pretreatment: An alternative in second-generation ethanol from wheat straw. Bioresource Technology, v. 102, n. 16, p. 7500-7506, 2011.

SINDHU, R.; BINOD, P.; PANDEY, A. Biological pretreatment of lignocellulosic biomass An overview. Bioresource Technology, v. 199, p. 76-82, 2016.

UMMALYMA, S. B. et al. Biological pretreatment of lignocellulosic biomass-current trends and future perspectives. [s.l.] Elsevier Inc., 2019.

VAN VLEET, Jh; JEFFRIES, Tw. Yeast metabolic engineering for hemicellulosic ethanol production. Current Opinion In Biotechnology, Madison, v. 20, n. 3, p. 300-306, jul. 2009.

VOHRA, M. et al. Bioethanol production: Feedstock and current technologies. Journal of Environmental Chemical Engineering, v. 2, n. 1, p. 573-584, 2014.

WAN, C.; LI, Y. Fungal pretreatment of lignocellulosic biomass. Biotechnology Advances, v. 30, n. 6, p. 1447-1457, 2012.

WANDERLEY, M. C. DE A. et al. Increase in ethanol production from sugarcane bagasse based on combined pretreatments and fed-batch enzymatic hydrolysis. Bioresource Technology, v. 128, p. 448-453, 2013.

WATKINS, D. et al. Extraction and characterization of lignin from different biomass resources. Integrative Medicine Research, v. 4, n. 1, p. 26-32, 2014.

ZABED, H. et al. Fuel ethanol production from lignocellulosic biomass: An overview on feedstocks and technological approaches. Renewable and Sustainable Energy Reviews, v. 66, p. 751-774, 2016.

ZABED, H. et al. Bioethanol production from renewable sources: Current perspectives and technological progress. Renewable and Sustainable Energy Reviews, v. 71, n. October 2015, p. 475-501, 2017. 